\title{
Meta Distribution of Downlink SIR for Binomial Point Processes
}

\author{
Nour Kouzayha, Member, IEEE, Hesham ElSawy, Senior Member, IEEE, Hayssam Dahrouj, Senior \\ Member, IEEE, and Tareq Y. Al-Naffouri, Senior Member, IEEE
}

\begin{abstract}
The meta distribution (MD) of the signal to interference ratio (SIR) extends stochastic geometry analysis from spatial averages to reveals find-grained information about the network performance. There have been several efforts to establish the MD framework for the Poisson point process (PPP) and other ergodic point processes. However, the MD analysis for finite point processes is overlooked. In this paper, we develop the MD of the binomial point process (BPP), which is practical for cases with a priori knowledge about the number of devices as well as their geographical spatial existence. For such finite models, we define the MD as a location-dependent likelihood of a receiver to achieve a required SIR with a probability more than a predefined threshold. The letter also extends the MD of the BPP to find the MD of finite PPP and verifies the convergence of the newly derived MD to the ergodic PPP's MD. The obtained analytical derivations are validated using Monte-Carlo simulations.
\end{abstract}

Index Terms-Binomial point process (BPP), meta distribution (MD), success probability (SP), finite PPP (FPPP), SIR, stochastic geometry.

\section{INTRODUCTION}

A CCURATE spatial modeling of wireless networks is critical to characterize their performance and obtain useful insights. Baseline network models have relied on using homogeneous infinite Poisson point processes (PPPs) [1]. Owing to its simplicity and analytical tractability, modeling the spatial distribution of transmitters or receivers with PPP is becoming more prominent, leading to useful insights about network performance. The conventional stochastic geometry analysis of wireless networks relies on spatial averages of success (coverage) probability (SP). However, spatial averages do not reveal how the SP varies across the network nor the percentiles of users that meet specific performance benchmarks. Recently, the meta distribution (MD) of the signal to interference ratio (SIR) is proposed in [2] as a new key performance metric that offers fine-grained information about the network. The MD is important to characterize delay in large-scale networks, where the devices locations remain static for large number of packets transmissions and fading realizations [3].

Despite the accuracy of PPP in modeling large scale networks, it is not quite suitable to model a finite network, constituted of a fixed number of nodes that are located within a given finite area. For such networks, the binomial point process (BPP) can be used as a simple and reasonable model for the spatial distribution of the existing nodes [4]. While the MD of the DL SIR has been thoroughly investigated for PPP-based networks and more generally for any ergodic point

N. Kouzayha, H. Dahrouj, and T. Y. Al-Naffouri, are with the Division of Computer, Electrical and Mathematical Sciences and Engineering, King Abdullah University of Science and Technology, Thuwal, Saudi Arabia (e-mail: nour.kouzayha@kaust.edu.sa; hayssam.dahrouj@kaust.edu.sa; tareq.alnaffouri@kaust.edu.sa).

H. ElSawy is with the Electrical Engineering Department, King Fahd University of Petroleum and Minerals (KFUPM), Dhahran, Saudi Arabia. (email: hesham.elsawy@kfupm.edu.sa). process, this characterization for the finite BPP model is not yet established, which is the primary purpose of this letter.

The MD of non-PPP networks has been already addressed in few recent works [5], [6]. In particular, the work in [5] utilizes the deployment gain to obtain approximate MD for general stationary and ergodic point processes. The work in [6] focuses on the MD in Poisson cluster point process, which is an important special case of stationary and ergodic point processes. The MD is meant to capture the variability in the long-term SP under static network realization and varying fading and devices activities. For ergodic point processes, the following is true for the MD: (i) The MD captures the SP variability for a given location (e.g., origin) across different networks realizations. Such MD is location-independent due to the translation invariance of the point process, (ii) Since the ensemble average is equivalent to the spatial average, the MD intrinsically captures the percentile performance of different devices within an arbitrary realization of the network.

For the finite BPP, both aforementioned facts are not true. Compared to PPP, BPP is neither ergodic nor stationary, and the devices performance is location-dependent. For instance, the aggregate interference and the SIR of a receiver located at the center of the network differ significantly from a receiver at the network edge. In addition, the distances from the transmitters to an arbitrary located receiver are correlated because of the mutual distance between the receiver and the center of the network. Thus, the MD of the BPP captures the performance variability for a given location across different networks realizations. Since the BPP is finite and isotropic, the MD is location-dependent in the sense that the location is measured in terms of the distance from the origin. For the design of BPP networks, the MD gives insights on the likelihood of the devices to achieve a required SP as function of their relative locations from the network center.

To this end, this letter characterizes the location-dependent SIR MD of a BPP, which finds applications in networks with a priori knowledge about the number of devices as well as their spatial existence. Such MD is also critical for latency characterization and spatio-temporal analysis, which assume static networks for long time duration. As the MD exact expression is computationally infeasible, the moments are used to obtain a close approximation using the beta distribution. Furthermore, the letter provides the MD of a finite PPP and shows that, as the region becomes large, this MD converges to the infinite PPP's MD. Monte-Carlo simulations are conducted to prove the accuracy of the analytical framework.

\section{SySTEM MODEL}

A downlink (DL) finite wireless network is considered where a fixed number of transmitters $N$ are deployed in a 
bounded area to serve the receivers. The locations of the transmitters are modeled as a uniform BPP in a finite region $\mathcal{A} \subset \mathbb{R}^{2}$. We consider that $\mathcal{A}=\mathbf{b}\left(\mathbf{o}, r_{d}\right)$ is a disk of radius $r_{d}$ centered around the origin $\mathbf{o}=(0,0)$. All the transmitters, located at $\left\{\mathbf{y}_{t}\right\}_{t=1: N} \equiv \Phi$, are active independently with probability $p$ and transmit with unit power in each time slot. The analysis is performed for a reference receiver at an arbitrary location $\mathbf{v}_{0}$ from the origin. Since uniform BPP in $\mathbf{b}\left(\mathbf{o}, r_{d}\right)$ is isotropic and rotation invariant around the origin, we assume that the $\mathrm{x}$-axis is aligned with the location of the reference receiver such that $\mathbf{v}_{0}=\left(x_{0}, 0\right)$, where $\left\|\mathbf{v}_{0}\right\|=x_{0} \in\left[0, r_{d}\right]$ and $\|\cdot\|$ is the Euclidean norm. We assume that the receiver associates with the nearest transmitter located at $\mathbf{y}_{s} \triangleq \operatorname{argmin}\left\{\mathbf{y}_{t} \in \Phi:\left\|\mathbf{y}_{t}-\mathbf{v}_{0}\right\|\right\}$ to get service, and so the remaining $N-1$ transmitters are considered interferers. We next provide the distance distributions between the receiver and an arbitrary transmitter, the nearest transmitter, and an interfering transmitter, similar to the approach adopted in [4]. Thus, the probability distribution function (PDF) of the distance separating the receiver from an arbitrary transmitter from the set of independently and identically distributed (i.i.d.) transmitters $\left\{\mathbf{y}_{t}\right\}_{t=1: N}$, conditioned on $x_{0}=\left\|\mathbf{v}_{0}\right\|$, is

$$
f_{W}(w)= \begin{cases}f_{W_{1}}(w), & 0 \leq w \leq w_{m} \\ f_{W_{2}}(w), & w_{m} \leq w \leq w_{p}\end{cases}
$$

where $f_{W_{1}}(w)=\frac{2 w}{r_{d}^{2}}, f_{W_{2}}(w)=\frac{2 w}{\pi r_{d}^{2}} \arccos \left(\frac{w^{2}+x_{0}^{2}-r_{d}^{2}}{2 x_{0} w}\right)$, $w_{m}=r_{d}-x_{0}$ and $\stackrel{d}{w}_{p}=r_{d}+x_{0}$. The PDF of the distance $R=\left\|\mathbf{v}_{0}-\mathbf{y}_{s}\right\|$ between the receiver and the nearest transmitter located at $\mathbf{y}_{s}$, to which it associates, is obtained by referring to the conditionally i.i.d. transmitters' locations as in [4]

$$
f_{R}(r)= \begin{cases}f_{R_{1}}(r), & 0 \leq r \leq w_{m} \\ f_{R_{2}}(r), & w_{m} \leq r \leq w_{p}\end{cases}
$$

where $f_{R_{1}}(r)=N f_{W_{1}}(r)\left(1-F_{W_{1}}(r)\right)^{N-1}$ and $f_{R_{2}}(r)=$ $N f_{W_{2}}(r)\left(1-F_{W_{2}}(r)\right)^{N-1}$. Here, $F_{W_{1}}(\cdot)$ and $F_{W_{2}}(\cdot)$ represent the piecewise cumulative distribution function (CDF) of the distance from the receiver to an arbitrary transmitter. Finally, the PDF of the distance from the receiver to an arbitrary interferer from the unordered set of $(N-1)$ i.i.d transmitters is given by conditioning on the distance to the nearest transmitter $r$ as

$$
f_{U}(u)= \begin{cases}\frac{f_{W_{1}}(u)}{1-F_{W_{1}}(r)}, & 0 \leq r \leq w_{m} ; r \leq u \leq w_{p} \\ \frac{f_{W_{2}}(u)}{1-F_{W_{1}}(r)}, & 0 \leq r \leq w_{m} ; w_{m} \leq u \leq w_{p} \\ \frac{f_{W_{2}}(u)}{1-F_{W_{2}}(r)}, & w_{m} \leq r \leq w_{p} ; r \leq u \leq w_{p},\end{cases}
$$

where $f_{W_{1}}(\cdot)$ and $f_{W_{2}}(\cdot)$ are given in 11 and $F_{W_{1}}(\cdot)$ and $F_{W_{2}}(\cdot)$ are the corresponding CDFs.

The signal propagation experiences small scale Rayleigh fading with exponential distribution and unit mean and distance dependent path-loss. The path-loss is modeled as $l(\mathbf{x})=$ $\|\mathbf{x}\|^{-\alpha}$, where $\alpha>2$ is the path-loss exponent. The SIR experienced by the reference receiver at $\mathbf{v}_{0}$ is

$$
\operatorname{SIR}\left(x_{0}\right)=\frac{h_{s}\left\|\mathbf{v}_{0}-\mathbf{y}_{s}\right\|^{-\alpha}}{\sum_{\mathbf{y}_{t} \in \Phi \backslash \mathbf{y}_{s}} a_{t} h_{t}\left\|\mathbf{v}_{0}-\mathbf{y}_{t}\right\|^{-\alpha}},
$$

where $\mathbf{y}_{s}$ and $\mathbf{y}_{t}$ are the locations of the serving transmitter and $t$-th transmitter, $h_{s}$ and $h_{t} \sim \exp (1)$ model the small scale
Rayleigh fading. $\sum_{\mathbf{y}_{t} \in \Phi \backslash \mathbf{y}_{s}} a_{t} h_{t}\left\|\mathbf{v}_{0}-\mathbf{y}_{t}\right\|^{-\alpha}$ is the interference from the remaining $N-1$ transmitters and $a_{t} \sim \operatorname{Bernoulli}(p)$ denotes the $t$-th interfering transmitter activity.

\section{SIR META DistRIBUTION FOR BPP}

The general expression of the SIR MD for a given SIR threshold $\theta$ and reliability level $\gamma$ is given in [2] as

$$
\bar{F}\left(\theta, x_{0}, \gamma\right) \triangleq \mathbb{P}\left(P_{s}\left(\theta, x_{0}\right)>\gamma\right), \theta \in \mathbb{R}^{+}, \gamma \in[0,1],
$$

where $P_{s}\left(\theta, x_{0}\right)$ is the SP conditioned on the point process $\Phi$ of transmitters when the receiver is at distance $x_{0}$ from the origin, i.e.

$$
P_{s}\left(\theta, x_{0}\right)=\mathbb{P}\left(\operatorname{SIR}\left(x_{0}\right)>\theta \mid \Phi\right) .
$$

The probability in (6) is a function of the receiver's location $x_{0}$ and is computed by averaging over the small scale fading and the transmitters activity. The MD is thus the complementary cumulative distribution function (CCDF) of the conditional SP $P_{s}\left(\theta, x_{0}\right)$. It is worth reemphasizing that the percentile performance interpretation of the MD does not hold for BPP. Instead, the MD of BPP captures the SP distribution across different static realizations of the BPP.

\section{A. Exact Meta Distribution and Moments Calculation}

As aforementioned, the $\mathrm{MD}$ is the $\mathrm{CCDF}$ of the conditional SP $P_{s}\left(\theta, x_{0}\right)$. While determining the distribution of $P_{s}\left(\theta, x_{0}\right)$ initially looks intricate, using the Gil-Pelaez approach with imaginary moments, an exact expression of the MD can be obtained [2]. The $b$-th moment $M_{b}$ of the SP $P_{s}\left(\theta, x_{0}\right)$ given that the receiver is at distance $x_{0}$ from the origin is defined as

$$
M_{b} \triangleq \mathbb{E}\left[P_{s}\left(\theta, x_{0}\right)^{b}\right], b \in \mathbb{C} .
$$

These moments can be used to extract the exact expression of the MD using the Gil-Pelaez theorem as follows

$$
\bar{F}\left(\theta, x_{0}, \gamma\right)=\frac{1}{2}+\frac{1}{\pi} \int_{0}^{\infty} \frac{\operatorname{Im}\left[e^{-\mathrm{j} t \log x} M_{\mathrm{j} t}\right]}{t} \mathrm{~d} t, \gamma \in[0,1],
$$

where $\operatorname{Im}[\cdot]$ denotes the imaginary part of a complex number and $M_{\mathrm{j} t}$ is the moment of $P_{s}\left(\theta, x_{0}\right)$ for $b=j t$.

Theorem 1. For a receiver located at a distance $x_{0}$ from the origin of a disk of radius $r_{d}$, the b-th moment of the conditional $S P P_{s}\left(\theta, x_{0}\right)$ is given by (10) at the top of next page where $N$ is the transmitters' number, $\theta$ is the SIR threshold, and $\alpha$ is the path-loss exponent. $f_{W_{1}}(\cdot)$ and $f_{W_{2}}(\cdot)$ are defined in (1).

Proof: The conditional SP $P_{s}\left(\theta, x_{0}\right)$ is given by

$$
\begin{aligned}
P_{s}\left(\theta, x_{0}\right) & \stackrel{(a)}{=} \mathbb{P}\left[h_{s}>\theta r^{\alpha} \sum_{t=1}^{N-1} a_{t} h_{t} u_{t}^{-\alpha}\right] \\
& \stackrel{(b)}{=} \mathbb{E}_{h_{t}}\left[\exp \left(-\theta r^{\alpha} \sum_{t=1}^{N-1} a_{t} h_{t} u_{t}^{-\alpha}\right)\right] \\
& \stackrel{(c)}{=} \prod_{t=1}^{N-1}\left(\frac{p}{1+\theta\left(r / u_{t}\right)^{\alpha}}+1-p\right)
\end{aligned}
$$

Here, (a) follows from substituting (4) in (6) and rearranging where for notational convenience, we define $r \triangleq\left\|\mathbf{v}_{0}-\mathbf{y}_{s}\right\|$ and $u_{t} \triangleq\left\|\mathbf{v}_{0}-\mathbf{y}_{t}\right\|$, (b) follows by involving the exponential distribution of $h_{s}$, and (c) is obtained by averaging over the 


$$
\begin{aligned}
& M_{b}=N \int_{0}^{w_{m}} r^{N-1} f_{W_{1}}(r)\left(\int_{\frac{r}{w_{m}}}^{1}\left(\frac{p}{1+\theta x^{\alpha}}+1-p\right)^{b} f_{W_{1}}\left(\frac{r}{x}\right) x^{-2} \mathrm{~d} x+\int_{\frac{r}{w_{p}}}^{\frac{r}{w_{m}}}\left(\frac{p}{1+\theta x^{\alpha}}+1-p\right)^{b} f_{W_{2}}\left(\frac{r}{x}\right) x^{-2} \mathrm{~d} x\right)^{N-1} \mathrm{~d} r \\
& +N \int_{w_{m}}^{w_{p}} r^{N-1} f_{W_{2}}(r)\left(\int_{\frac{r}{w_{p}}}^{1}\left(\frac{p}{1+\theta x^{\alpha}}+1-p\right)^{b} f_{W_{2}}\left(\frac{r}{x}\right) x^{-2} \mathrm{~d} x\right)^{N-1} \mathrm{~d} r
\end{aligned}
$$

$M_{b}^{0}=2 N$

$\int_{0}^{1} x^{2 N-1}\left(\sum_{k=0}^{b}\left(\begin{array}{l}b \\ k\end{array}\right) \frac{(-p \theta)^{k}}{1-\frac{k}{\delta}}\left[x^{-2\left(1-\frac{k}{\delta}\right)}\left(1+\theta x^{\frac{2}{\delta}}\right)^{-k}{ }_{2} F_{1}\left(k, 1 ; 1+k-\delta ; \frac{1}{1+\frac{1}{\theta} x^{-\frac{2}{\delta}}}\right)-(1+\theta)^{-k}{ }_{2} F_{1}\left(k, 1 ; 1+k-\delta ; \frac{\theta}{1+\theta}\right)\right]\right)^{N-1} \mathrm{~d} x$,

i.i.d. fading gains $h_{t}$ and the transmitters' activity $a_{t}$, 2 . Appendix A]. We can use this expression for $P_{s}\left(\theta, x_{0}\right)$ to evaluate the corresponding $b$-th moment as

$$
\begin{aligned}
& M_{b}=\mathbb{E}\left[\prod_{t=1}^{N-1}\left(\frac{p}{1+\theta\left(r / u_{t}\right)^{\alpha}}+1-p\right)^{b}\right]= \\
& \int_{0}^{w_{p}}\left(\int_{r}^{w_{p}}\left(\frac{p}{1+\theta(r / u)^{\alpha}}+1-p\right)^{b} f_{U}(u) \mathrm{d} u\right)^{N-1} f_{R}(r) \mathrm{d} r .
\end{aligned}
$$

The expectation in (12) is evaluated in two steps. Firstly, average over the distance to the interferer $u$ conditioned on the distance to the serving transmitter $r$, and secondly average the result over $r$. Here, $f_{R}(\cdot)$ and $f_{U}(\cdot)$ are the PDFs of the distances $r$ and $u$, as illustrated in (2) and (3), respectively. Lastly, by replacing $r / u$ with $x$, we can get $(10)$.

Corollary 1. When the reference receiver is placed at the origin $\left(x_{0}=0\right)$, the b-th moment $M_{b}^{0}$ of the conditional $S P$ simplifies to (11), where $\delta=2 / \alpha$ and ${ }_{2} F_{1}(a, b ; c ; x)=$ $\sum_{n=0}^{\infty} \frac{(a)_{n}(b)_{n}}{(c)_{n}} \frac{x^{n}}{n !}$ is the Gauss hypergeometric function.

Proof: The $b$-th moment of the SP for a receiver located at the origin $\left(x_{0}=0\right)$ is derived as

$$
\begin{aligned}
& M_{b}^{0}=N \int_{0}^{r_{d}} r^{N-1} f_{W_{1}}(r) \\
& \left(\int_{\frac{r}{r_{d}}}^{1}\left(\frac{p}{1+\theta x^{\alpha}}+1-p\right)^{b} f_{W_{1}}\left(\frac{r}{x}\right) x^{-2} \mathrm{~d} x\right)^{N-1} \mathrm{~d} r=N \\
& \left(\frac{2}{r_{d}^{2}}\right)^{N} \int_{0}^{r_{d}} r^{2 N-1}\left(\int_{\frac{r}{r_{d}}}^{1} x^{-3}\left(\frac{p}{1+\theta x^{\alpha}}+1-p\right)^{b} \mathrm{~d} x\right)^{N-1} \mathrm{~d} r,
\end{aligned}
$$

where we use the expression for $f_{W_{1}}(\cdot)$ of $(1)$. Using the change of variable $y=x^{\alpha}$ and the binomial expansion, we expand the inner integral in $(13)$ as

$$
\begin{aligned}
& \int_{\frac{r}{r_{d}}}^{1} x^{-3}\left(\frac{p}{1+\theta x^{\alpha}}+1-p\right)^{b} \mathrm{~d} x \\
& =\frac{1}{\alpha} \sum_{k=0}^{b}\left(\begin{array}{l}
b \\
k
\end{array}\right)(-p \theta)^{k} \int_{\left(\frac{r}{r_{d}}\right)^{\alpha}}^{1} \frac{y^{k-\frac{2}{\alpha}-1}}{(1+\theta y)^{k}} \mathrm{~d} y=\frac{1}{\alpha} \sum_{k=0}^{b}\left(\begin{array}{l}
b \\
k
\end{array}\right)(-p \theta)^{k} \\
& \left(\int_{0}^{1} \frac{y^{k-\frac{2}{\alpha}-1}}{(1+\theta y)^{k}} \mathrm{~d} y-\int_{0}^{\left(\frac{r}{r_{d}}\right)^{\alpha}} \frac{y^{k-\frac{2}{\alpha}-1}}{(1+\theta y)^{k}} \mathrm{~d} y\right) \\
& =\frac{1}{2} \sum_{k=0}^{b}\left(\begin{array}{l}
b \\
k
\end{array}\right)(-p \theta)^{k}\left(-\frac{1}{1-\frac{k}{\delta}} F_{1}(k, k-\delta ; 1+k-\delta ;-\theta)\right. \\
& \left.+\frac{1}{1-\frac{k}{\delta}}\left(\frac{r}{r_{d}}\right)^{-2\left(1-\frac{k}{\delta}\right)}{ }_{2} F_{1}\left(k, k-\delta ; 1+k-\delta ;-\theta\left(\frac{r}{r_{d}}\right)^{\frac{2}{\delta}}\right)\right),
\end{aligned}
$$

which follows from the fact that $\int_{0}^{u} \frac{x^{\mu-1}}{(1+\beta x)^{\nu}} \mathrm{d} x=$ $\frac{u^{\mu}}{\mu}{ }_{2} F_{1}(\nu, \mu ; 1+\mu ;-\beta u)$ [7, p.315]. By plugging [14 in [13 and using the variable substitution $x=\frac{r}{r_{d}}$, we get

$$
\begin{aligned}
& M_{b}^{0}=2 N \int_{0}^{1} x^{2 N-1}\left(\sum _ { k = 0 } ^ { b } ( \begin{array} { l } 
{ b } \\
{ k }
\end{array} ) \frac { ( - p \theta ) ^ { k } } { 1 - \frac { k } { \delta } } \left(x^{-2\left(1-\frac{k}{\delta}\right)}\right.\right. \\
& { }_{2} F_{1}\left(k, k-\delta ; 1+k-\delta ;-\theta x^{\frac{2}{\delta}}\right) \\
& \left.\left.-{ }_{2} F_{1}(k, k-\delta ; 1+k-\delta ;-\theta)\right)\right)^{N-1} \mathrm{~d} x .
\end{aligned}
$$

Finally, by using the Euler approximation of the hypergeometric function in (15), we can get the expression in (11).

\section{B. Approximation of Meta Distribution}

The MD of the conditional SP is difficult to obtain from (8). So we use the beta distribution to approximate it on its support $[0,1]$ [2]. This can be done by matching the first and second moments to the mean and variance of the beta distribution as follows

$$
\bar{F}\left(\theta, x_{0}, \gamma\right) \approx 1-\frac{1}{\mathrm{~B}\left(\mu_{1}, \mu_{2}\right)} \int_{0}^{\gamma} t^{\mu_{1}-1}(1-t)^{\mu_{2}-1} \mathrm{~d} t,
$$

where $\gamma \in[0,1], \mathrm{B}(a, b)$ is the beta function, $\mu_{1}=$ $\frac{M_{1}\left(M_{1}-M_{2}\right)}{M_{2}-M_{1}^{2}}, \mu_{2}=\frac{\left(M_{1}-M_{2}\right)\left(1-M_{1}\right)}{M_{2}-M_{1}^{2}}$, and $M_{1}$ and $M_{2}$ are the first and second moments of the conditional SP given in (10).

\section{Extension to PPP finite and infinite deployments}

While the MD of the SP for the PPP is widely adopted in the literature, only infinite deployments are considered. In this section, we first extend the BPP MD to find that of a finite PPP (FPPP). Then, we show the convergence to the well-known MD for ergodic PPP when we let the deployment area grow unboundedly. This 2-stage technique that we follow to obtain the limiting behavior of BPP is similar to [8]. To find the MD of the FPPP, we let the number of transmitters $N$ to randomly vary across different realizations according to a Poisson distribution with mean $\lambda \pi r_{d}^{2}$ and derive the $b$ th moment of the conditional SP of a FPPP as given in the following lemma.

Lemma 1. The b-th moment of the conditional SP of a FPPP with density $\lambda$ in a finite region of radius $r_{d}$ is given as

$$
\begin{aligned}
& M_{b}^{F P P P}= \\
& \exp \left(-\lambda \pi r_{d}^{2}\right) \int_{0}^{r_{d}}(2 \lambda \pi r) \exp \left(-\lambda \pi r^{2}{ }_{2} F_{1}(b,-\delta ; 1-\delta ;-\theta)\right. \\
& \left.+\lambda \pi r_{d 2}^{2} F_{1}\left(b,-\delta ; 1-\delta ;-\theta\left(\frac{r}{r_{d}}\right)^{\frac{2}{\delta}}\right)\right) \mathrm{d} r .
\end{aligned}
$$


where $\delta=2 / \alpha$ and ${ }_{2} F_{1}(a, b ; c ; x)$ is the Gauss hypergeometric function.

$$
\begin{aligned}
& \text { Proof: For } x_{0}=0 \text { and } p=1,(12) \text { reduces to } \\
& M_{b}^{\mathrm{BPP}}=k \int_{0}^{r_{d}} \frac{2 r}{r_{d}^{2}}\left(\int_{r}^{r_{d}} \frac{1}{\left(1+\theta\left(\frac{r}{u}\right)^{\alpha}\right)^{b}} \frac{2 u}{r_{d}^{2}} \mathrm{~d} u\right)^{k-1} \mathrm{~d} r .
\end{aligned}
$$

This expression is conditioned on having $k$ transmitters in the disk $\mathbf{b}\left(\mathbf{o}, r_{d}\right)$. The $b$-th moment of the FPPP is obtained in (19) by deconditioning on $k$ assumed to have a Poisson distribution.

$$
\begin{aligned}
& M_{b}^{\mathrm{FPP}}=\mathbb{E}\left[M_{b}^{\mathrm{BPP}} \mid \Phi\left(\mathbf{b}\left(\mathbf{o}, r_{d}\right)\right)=k\right] \\
& =\mathbb{E}\left[k \int_{0}^{r_{d}} \frac{2 r}{r_{d}^{2}}\left(\int_{r}^{r_{d}} \frac{1}{\left(1+\theta\left(\frac{r}{u}\right)^{\alpha}\right)^{b}} \frac{2 u}{r_{d}^{2}} \mathrm{~d} u\right)^{k-1} \mathrm{~d} r\right. \\
& \left.\mid \Phi\left(b\left(0, r_{d}\right)\right)=k\right] .
\end{aligned}
$$

The probability of finding $k$ transmitters in $\mathbf{b}\left(\mathbf{o}, r_{d}\right)$ can be obtained using the Poisson distribution. Denoting $f\left(r, r_{d}\right)=$ $\int_{r}^{r_{d}} \frac{1}{\left(1+\theta\left(\frac{r}{u}\right)^{\alpha}\right)^{b}} \frac{2 u}{r_{d}^{2}} \mathrm{~d} u$, we get

$$
\begin{aligned}
& M_{b}^{\mathrm{FPPP}}=\sum_{k=0}^{\infty} \exp \left(-\lambda \pi r_{d}^{2}\right) \frac{\left(\lambda \pi r_{d}^{2}\right)^{k}}{k !} k \int_{0}^{r_{d}} \frac{2 r}{r_{d}^{2}} f\left(r, r_{d}\right)^{k-1} \mathrm{~d} r \\
& \stackrel{(a)}{=} \exp \left(-\lambda \pi r_{d}^{2}\right) \int_{0}^{r_{d}} \frac{2 r}{r_{d}^{2}} \sum_{k=0}^{\infty} k\left(\lambda \pi r_{d}^{2}\right) \frac{\left(\lambda \pi r_{d}^{2}\right)^{k-1}}{k !} f\left(r, r_{d}\right)^{k-1} \mathrm{~d} r \\
& =\exp \left(-\lambda \pi r_{d}^{2}\right) \int_{0}^{r_{d}}(2 \lambda \pi r) \sum_{k=0}^{\infty} \frac{\left(\lambda \pi r_{d}^{2} f\left(r, r_{d}\right)\right)^{k-1}}{(k-1) !} \mathrm{d} r . \\
& \stackrel{(b)}{=} \exp \left(-\lambda \pi r_{d}^{2}\right) \int_{0}^{r_{d}}(2 \lambda \pi r) \exp \left(\lambda \pi r_{d}^{2} f\left(r, r_{d}\right)\right) \mathrm{d} r .
\end{aligned}
$$

where $\lambda$ is the density of the FPPP and (a) is obtained by inverting the order of the summation and the integration, (b) follows from summing over $k$ and interpreting the sum as the Taylor expansion of the exponential function $\sum_{k=0}^{\infty} \frac{x^{k}}{k !}=e^{x}$. Finally, by applying first the substitution $x=\frac{r}{u}$ and then the substitution $y=x^{\alpha}$ in $f\left(r, r_{d}\right)$, we obtain

$$
\begin{aligned}
& f\left(r, r_{d}\right)=\frac{2 r^{2}}{r_{d}^{2}} \frac{1}{\alpha} \int_{\left(\frac{r}{r_{d}}\right)^{\alpha}}^{1} \frac{y^{-\frac{2}{\alpha}-1}}{(1+\theta y)^{b}} \mathrm{~d} y \\
& =\frac{2 r^{2}}{r_{d}^{2}} \frac{1}{\alpha} \int_{0}^{1} \frac{y^{-\frac{2}{\alpha}-1}}{(1+\theta y)^{b}} \mathrm{~d} y-\frac{2 r^{2}}{r_{d}^{2}} \frac{1}{\alpha} \int_{0}^{\left(\frac{r}{r_{d}}\right)^{\alpha}} \frac{y^{-\frac{2}{\alpha}-1}}{(1+\theta y)^{b}} \mathrm{~d} y \\
& =-\frac{r^{2}}{r_{d}^{2}} 2 F_{1}(b,-\delta ; 1-\delta ;-\theta)+{ }_{2} F_{1}\left(b,-\delta ; 1-\delta ;-\theta\left(\frac{r}{r_{d}}\right)^{\frac{2}{\delta}}\right),
\end{aligned}
$$

which follows from [7, p.315] and $\delta=2 / \alpha$. By plugging [21 in 20], we obtain the final expression in (17).

After deriving the $b$-th moment of the FPPP, we prove in Corollary 2 that such moment converges to the $b$-th moment of an infinite PPP when the considered area becomes large.

Corollary 2. If the radius of the finite area goes to infinity, the $b$-th moment of a FPPP $M_{b}^{F P P P}$ converges to the b-th moment of an infinite $P P P M_{b}^{P P P}$

Proof: By letting $r_{d}$ go to $\infty$ we obtain,

$$
\begin{aligned}
& \lim _{r_{d} \rightarrow \infty} M_{b}^{\mathrm{FPPP}} \\
& =\lim _{r_{d} \rightarrow \infty} \int_{0}^{r_{d}}(2 \lambda \pi r) \exp \left(-\lambda \pi r^{2}{ }_{2} F_{1}(b,-\delta ; 1-\delta ;-\theta)\right) \\
& \times \exp \left(\lambda \pi r_{d}^{2}\left(-1+{ }_{2} F_{1}\left(b,-\delta ; 1-\delta ;-\theta\left(\frac{r}{r_{d}}\right)^{\frac{2}{\delta}}\right)\right)\right) .
\end{aligned}
$$

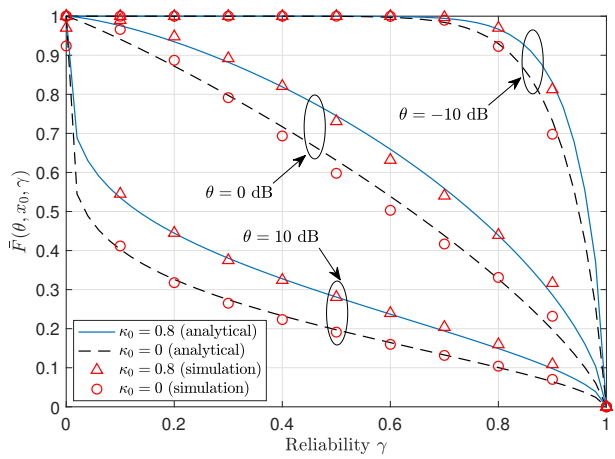

Fig. 1. Simulation and Beta approximation of the BPP MD for different values of $\theta$ and receiver normalized distance to origin $(p=1)$.

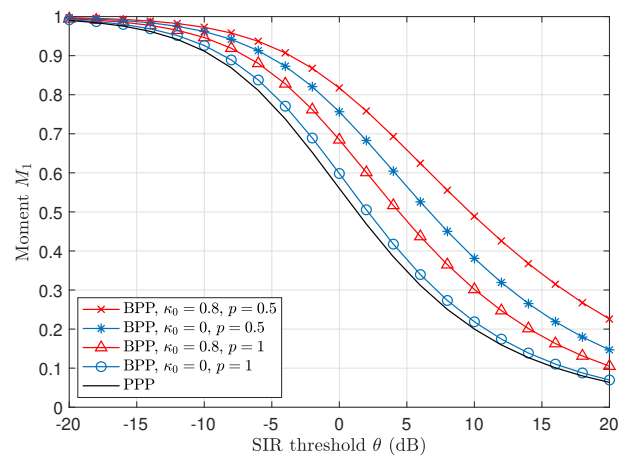

Fig. 2. Moment $M_{1}$ as function of $\theta$ for different $p$ and $\kappa_{0}$ values.

By applying the series expansion for ${ }_{2} F_{1}(a, b ; c ; x)$ at $x=0$, and the fact that $\frac{r}{r_{d}}$ goes to 0 as $r_{d} \rightarrow \infty$, we find that

$$
\lim _{r_{d} \rightarrow \infty}{ }_{2} F_{1}\left(b,-\delta ; 1-\delta ;-\theta\left(\frac{r}{r_{d}}\right)^{\frac{2}{\delta}}\right)=1 .
$$

Thus, the expression in 22) can be simplified to

$$
\lim _{r_{d} \rightarrow \infty} \int_{0}^{r_{d}} 2 \lambda \pi r \exp \left(-\lambda \pi r^{2}{ }_{2} F_{1}(b,-\delta ; 1-\delta ;-\theta)\right) \mathrm{d} r .
$$

By applying the variable substitution $x=\lambda \pi r^{2}$, we can get

$$
\lim _{r_{d} \rightarrow \infty} M_{b}^{\mathrm{FPPP}}=\frac{1}{{ }_{2} F_{1}(b,-\delta ; 1-\delta ;-\theta)}=M_{b}^{\mathrm{PPP}},
$$

which is the expression obtained in [2] for the $b$-th moment of the conditional SP of a DL cellular PPP network with nearest base station association and path-loss exponent $\alpha$.

\section{NUMERICAL RESULTS}

In this section, we verify the accuracy of the analytical analysis and approximation with Monte-Carlo simulations and highlight the impact of different system parameters. Since the serving and the interfering transmitters are selected from one BPP, their positions from the origin, are scaled with the same factor when we vary the radius $r_{d}$. This implies that the SIR and the MD of the reference receiver located at $\mathbf{v}_{0}=\left(x_{0}, 0\right)$ where $x_{0}=\kappa_{0} r_{d}$ and $\kappa_{0} \in[0,1]$, are independent of the choice of $r_{d}$ for a given $\kappa_{0}$. Thus, without loss of generality, $r_{d}$ is normalized to 1 . Unless otherwise stated, the number of transmitters $N$, the transmit power, the activity factor $p$, and the path-loss exponent $\alpha$ are set to $10,1 \mathrm{~W}, 1$ and 4 , respectively. Two different locations are 


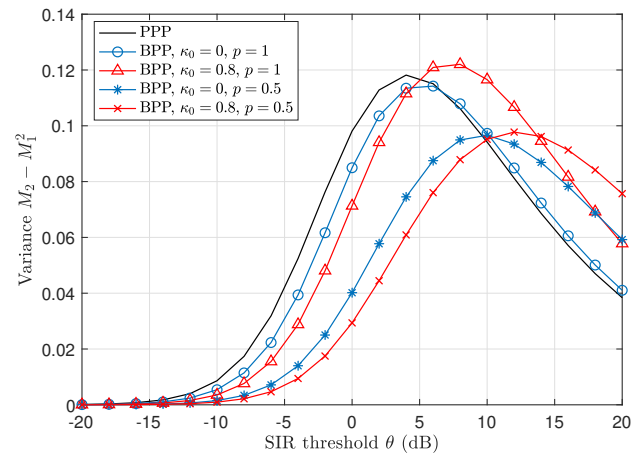

Fig. 3. Variance $M_{2}-M_{1}^{2}$ as function of $\theta$ for different $p$ and $\kappa_{0}$ values.

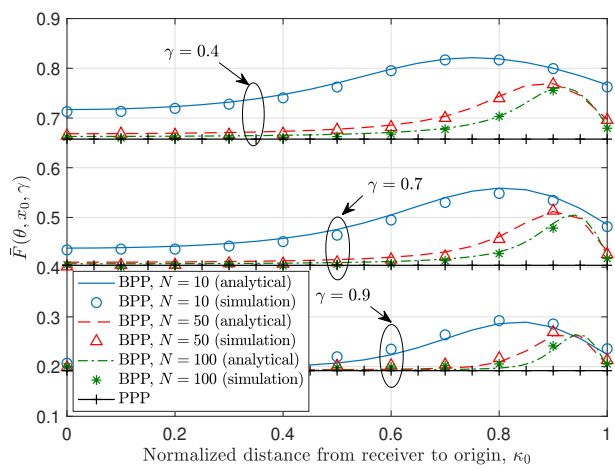

Fig. 4. MD as function of the reference receiver normalized distance to origin for PPP and BPP networks $(p=1, \theta=0 \mathrm{~dB})$.

considered for the reference receiver corresponding to $\kappa_{0}=0$ (receiver at the origin), and $\kappa_{0}=0.8$. Independent fading is considered for the intended link and the interfering links with exponential distribution and unit mean. The Monte-Carlo simulations construct the MD $\bar{F}\left(\theta, x_{0}, \gamma\right)$ across 5000 different BPP realizations with $10^{5}$ iterations per realization.

Fig. 1 presents the simulation (markers) and analytical (solid lines) results of the MD $\bar{F}\left(\theta, x_{0}, \gamma\right)$ for BPP as a function of the reliability $\gamma$ for different values of the SIR threshold, $\theta=-10 \mathrm{~dB}, \theta=0 \mathrm{~dB}$ and $\theta=10 \mathrm{~dB}$ and for two receiver locations. The close match between the simulation and the analytical results validates the developed model for the location-dependent MD of BPP and confirms the accuracy of the beta approximation. From Fig. 1, we note that, when the receiver is at a normalized distance $\kappa_{0}=0.8$ away from the origin, the likelihood that it has a SP of 0.7 across all the BPP realizations is about $20 \%$ (when $\theta=10 \mathrm{~dB}$ ), $54 \%$ (when $\theta=0 \mathrm{~dB}$ ), and $99 \%$ (when $\theta=-10 \mathrm{~dB}$ ). This likelihood value is even lower for a receiver at the center of the network due to the higher impact of interference.

Fig. 2 and Fig. 3 illustrate the SP reflected respectively by the first moment $M_{1}$ of the conditional SP and its variance $M_{2}-M_{1}^{2}$ as function of $\theta$ for different values of the transmitters' activity factor and for two receiver locations. In addition, the BPP results are compared against the moment and variance of a PPP whose moment expression is given in 25]. Fig. 2 shows that the SP of a randomly located receiver is higher than that of the receiver at the origin. This is due to the effect of the network boundary that experiences lower interference as compared to the network center. Fig. 3 shows that the variance of the conditional SP is also location-dependent. While the variance decays to zero for $\theta \rightarrow 0$ and $\theta \rightarrow \infty$, it has a maximum at a finite value of the SIR threshold. Fig. 2 and Fig. 3 confirm again the necessity of analyzing the MD of BPP networks and show that the spatially averaged SP increases, and the variance decreases, as we decrease the activity factor. Furthermore, by increasing $p$, the number of active transmitters increases and the gap between PPP and BPP results is reduced.

To better understand how the performance of a randomlylocated receiver differs from the central receiver, Fig. 4 plots the MD as a function of the distance from the reference receiver to the origin for different reliability values, $\gamma=0.4$, $\gamma=0.7$, and $\gamma=0.9$. Three different values are considered for the number of transmitters $N=10, N=50$ and $N=100$, and the obtained results are compared with the MD of a PPP determined from its moment expression given in (25). We notice that the BPP MD $\bar{F}\left(\theta, x_{0}, \gamma\right)$ increases slowly as the reference receiver moves away from the network center. This behavior is caused by the decrease of interference attributed to the network boundary effect of the finite BPP. As the receiver approaches the border of the considered area, the likelihood that this receiver gets covered with a given reliability level slips from the maximum level. Such reduction is because the interference reduction at the network edge is countered by a reduced likelihood to find a close by serving transmitter.

\section{CONCLUSION}

This letter characterizes the SIR MD of an arbitrary located receiver in a DL BPP network where the transmitters are distributed uniformly in a finite region. Due to the locationdependent feature of BPP, this MD provides the likelihood that a receiver satisfies the required performance across realizations instead of the percentiles performance of receivers. Among the letter's contributions are the precise derivation of the $b$ th moment of the conditional SP and the computation of an accurate approximation of the MD for BPP using the beta distribution. The letter also extends the MD of the BPP to find the MD of a finite PPP. To this end, the convergence to the MD of ergodic PPP is also presented, all together with the proper numerical validation of the analytical derivations.

\section{REFERENCES}

[1] A. Guo and M. Haenggi, "Spatial stochastic models and metrics for the structure of base stations in cellular networks," IEEE Trans. Wireless Commun., vol. 12, no. 11, pp. 5800-5812, 2013.

[2] M. Haenggi, "The meta distribution of the SIR in Poisson bipolar and cellular networks," IEEE Trans. Wireless Commun., vol. 15, no. 4, pp. 2577-2589, 2016.

[3] Y. Zhong, M. Haenggi, F. Zheng, W. Zhang, T. Q. S. Quek, and W. Nie, "Toward a tractable delay analysis in ultra-dense networks," IEEE Commun. Mag., vol. 55, no. 12, pp. 103-109, 2017.

[4] M. Afshang and H. S. Dhillon, "Fundamentals of modeling finite wireless networks using binomial point process," IEEE Trans. Wireless Commun., vol. 16, no. 5, pp. 3355-3370, 2017.

[5] S. S. Kalamkar and M. Haenggi, "Simple approximations of the SIR meta distribution in general cellular networks," IEEE Trans. Commun., vol. 67, no. 6, pp. 4393-4406, 2019.

[6] C. Saha, M. Afshang, and H. S. Dhillon, "Meta distribution of downlink SIR in a Poisson cluster process-based HetNet model," IEEE Wireless Commun. Lett., vol. 9, no. 12, pp. 2144-2148, 2020.

[7] "Definite integrals of elementary functions," in Table of Integrals, Series, and Products (Seventh Edition) (A. Jeffrey, D. Zwillinger, I. Gradshteyn, and I. Ryzhik, eds.), pp. 247 - 617, Boston: Academic Press, 2007.

[8] E. S. Sousa and J. A. Silvester, "Optimum transmission ranges in a direct-sequence spread-spectrum multihop packet radio network," IEEE J. Sel. Areas in Commun., vol. 8, no. 5, pp. 762-771, 1990. 